\title{
Low Cost UWB Printed Dipole Antenna with filtering feature
}

\author{
J.-F. Pintos $^{(1)}$, P. Chambelin ${ }^{(1)}$, A. Louzir ${ }^{(1)}$ and D. Rialet ${ }^{(2)}$, \\ (1) Thomson R\&D France, 1 Avenue Belle Fontaine, Cesson Sévigné, 35576, France \\ (2) IETR Institute of Electronics and Telecommunications of Rennes UMR-CNRS, \\ Université de Rennes I, Avenue du Général Leclerc, 35042 Rennes Cedex, France \\ jean-francois.pintos@thomson.net
}

\begin{abstract}
This paper introduces a new ultra-wideband (UWB) printed circular dipole antenna having a filtering feature for rejecting the Wi-Fi band (4.9-5.9 GHz). UWB regulation in Europe [1] and Japan require the rejection or the avoidance of the $5 \mathrm{GHz}$ Wi-Fi frequency band, but due to the very wide frequency band used by UWB technology, it is also particularly exposed to other systems disturbances. Some experiments revealed problems of coexistence between UWB and the local wireless networks working in the WLAN bands [2]. The proposed paper describes a Wi-Fi band rejection filter included in a previously developed compact UWB antenna [3]. Constraints such as the size and the cost of the antenna are taken into account in the design. Efficiency measurements of the realized filtering small antenna, using Schantz's method, are in conformance with simulations.
\end{abstract}

\section{Introduction}

At the present time UWB approach is another way to create high data rate links between devices. Such standard is based on very low power level over very large bandwidth (3.1-10.6 GHz). Nevertheless, in the WLAN band between 4.9 and 5.9 $\mathrm{GHz}$ the level of EIRP must be very low $(-70 \mathrm{dBm})$. This imposes to introduce filtering after or inside the antenna. Many solutions on frequency notched UWB antenna using the second approach with slots and/or arms in the antenna, have been proposed [4][5], but such solutions have generally poor level of rejection and narrow bandwidth. Our proposal is to introduce a stripline band reject filter in the low part of the antenna allowing improved Wi-Fi band rejection.

The stop band filter proposed is based on coupling open stubs and its form factor is imposed by the antenna shape.

The first part of this paper presents the design of such antenna with the filter included in the low part of the antenna.

The second part deals with simulated and measured results obtained in terms of S parameters and radiating performances. 


\section{Antenna Design and filter function}

The design is based on two arms (dipole like) with circular shapes (figure 1). The diameter of each arm is $0.20 \lambda$ (where $\lambda$ is the wavelength at the lowest frequency) which allows $S_{11}$ module to be lower than $-10 \mathrm{~dB}$ for frequencies higher than 3.1 $\mathrm{GHz}$. Two circular arms are printed on both faces of the substrate and linked in pairs with metallic holes located on the circumference of the arms. For simulation an FR4 substrate has been selected with $1 \mathrm{~mm}$ height, a relative permittivity $\mathcal{E}_{r}=4.4$ and $\tan \delta=0.02$. A 3 metallic layers substrate allows to feed the antenna by a stripline with ground planes corresponding to the 2 faces of the first circular arm. Thus, the feed line is hidden by the ground plane and a very "clean" radiation pattern is obtained (no spurious from fed line).

The filter can be then introduced in the feeding stripline part of the antenna (figure 2). The size of the selected filter is imposed by the antenna shape. It is based on five open stubs measuring approximately $\lambda_{\mathrm{g}} / 4$ each and separated from each other by an approximate distance of $\lambda_{\mathrm{g}} / 4$. Then, five short circuits are applied on the feed line. Moreover, some coupling effects are introduced between feed line and stubs to minimize the structure size.

\section{Simulated and experimental results}

From measurement results shown in Figure 3, the antenna is well matched from 3.1 to $4.5 \mathrm{GHz}$ and presents a return loss better the $-8 \mathrm{~dB}$ from 6.68 to $10.6 \mathrm{GHz}$. The previous design without the integrated filter [3], presents a return loss of -10 $\mathrm{dB}$ over all the UWB bandwidth.

The rejection appears very clearly on the Wi-Fi band [4.9 -5.9 GHz] on the simulated gain (figure 4) and on measured total efficiency based on Schantz's method well suited for small antennas (figure 6). A frequency shift of 100-200 $\mathrm{MHz}$ compared to simulations have been observed, that is why retro simulation has been conducted with new values of permittivity and loss tangent. The new substrate parameters have been obtained by measurement (not presented here). The retro simulation and the measurement are very similar from $\mathrm{S}$ parameters point of view.

Finally a comparison of the total antenna efficiency has been conducted in cases of using or not an integrated filter and between measurement and EM simulations (IE3D and HFSS). The measurements have been conducted in metallic sphere based on Schantz method [6].

The results show a quite good accordance between HFSS results and measurements and a bigger difference is obtained with IE3D (the required infinite substrate model under IE3D could explain such difference for high frequency band). The total efficiency of the antenna including the filter is higher than $50 \%$, while the antenna without filter (figure 5) was higher than $60 \%$. The difference is due to the insertion losses brought by the filter and which are estimated at $2 \mathrm{~dB}$. 


\section{Conclusion}

An ultra-wideband (UWB) printed circular dipole with filtering feature has been presented. Good performances have been obtained with this compact design close to expected values. This stand alone design is cheap, very easy to implement for classical mass production process and it allows simplification of the system for the DAA (Detection And Avoidance) implementation while ensuring an immunity of UWB devices against Wi-Fi systems.

\section{References}

[1] CEPT - ECC, "ECC Decision of dd mm 2006 on the harmonised conditions for devices using UWB technology in bands below 10.6 GHz", February 2006 draft (ECC/DEC/(06)AA), document 13_33-A8R0_Annex8_draft ECC Dec(06) AA_Modifications proposed by TG3.

[2] Hans G. Schantz, G. Wolenec, and Edward M. Myszka, "Frequency Notched UWB Antennas," IEEE conference on Ultra Wideband Systems and Technology, Nov. 2003

[3] E. Gueguen, F. Thudor, P. Chambelin, "A low cost UWB Printed Dipole Antenna with High Performances", Conference Proceedings of the 2005 IEEE International Conference on Ultra-Wideband, Zurich, Sept. 5-8, 2005

[4] Saou-Wen Su, Kin Lu Wong, fa Shian Chang, "Compact printed bandnotched ultra-wideband slot antenna", Antennas and Propagation Society International Symposium, 3-8 July 2005, pages 572- 575 vol. $2 B$

[5] Jianming Qiu, Zhengwei Du, Jianhua Lu, and Ke Gong, "A Planar Monopole Antenna Design With Band-Notched Characteristic", IEEE Transactions on Antennas and Propagation, vol. 54, No. 1, January 2006

[6] H.G. Schantz, "Measurement of UWB Antenna Efficiency", Vehicular Technology Conference, 2001, VTC 2001 Spring, IEEE VTS 53 rd, pages 1189-1191 vol.2

\section{Acknowledgments}

The authors wish to thank the French national agency for research (ANR) which has partially funded this work within the RNRT project AUBADE.

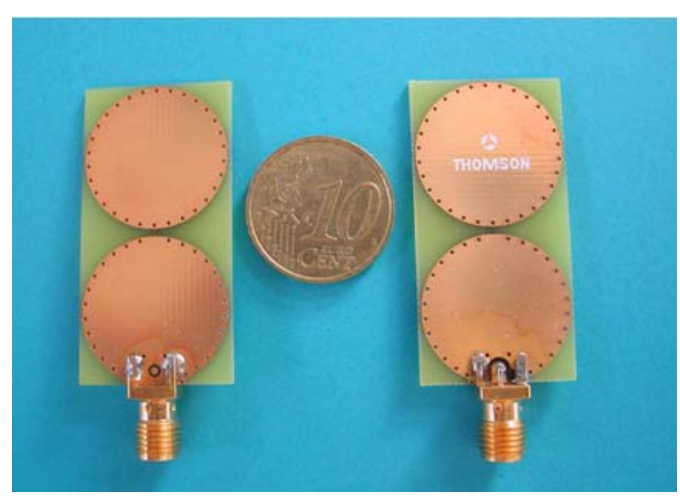

Figure 1 :View UWB antenna

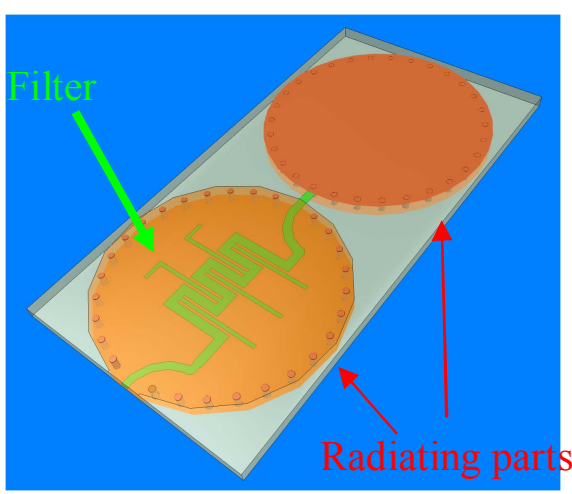

Figure 2 :View filter design 


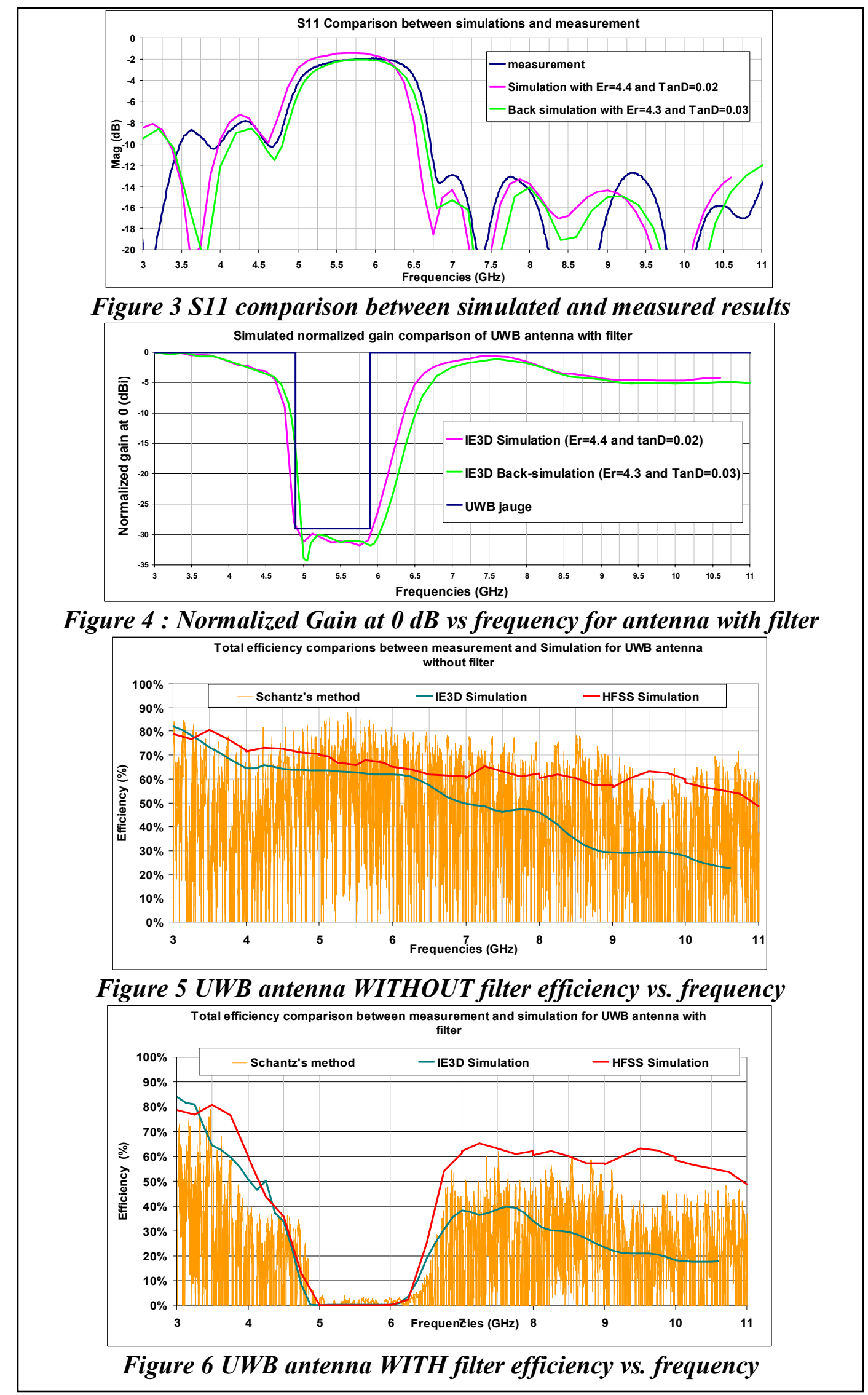

